\title{
A context-aware and social model of dynamic multiple criteria preferences
}

\author{
Evelina Giacchi ${ }^{1 *}$, Salvatore Corrente ${ }^{2}$, Alessandro Di Stefano ${ }^{1}$, Salvatore Greco ${ }^{2,3}$, Aurelio La Corte ${ }^{1}$ \\ and Marialisa Scatá ${ }^{1}$
}

*Correspondence:

evelina.giacchi@unict.it

${ }^{1}$ Department of Electric, Electronic and Computer

Engineering, University of Catania, Viale Andrea Doria 6, Cittadella Universitaria - Ed. Polifunzionale, 95127 Catania, Italy

Full list of author information is available at the end of the article

\begin{abstract}
We discuss a social decision making model in which individuals, represented by nodes, interact with each other through ties in a social network. Each node takes its decisions considering a set of points of view in a multiple criteria decision making perspective. Our model suggests the interplay of the following two features in the decision making process: the dynamic nature and the context-awareness of decisions. The dynamic nature is the result of the interaction among nodes producing a changing in preferences. The context-awareness, instead, represents the capability to take into account the knowledge background exploited by nodes to take their decisions. Thus, the two factors affecting the dynamics of preferences are: the inclination of each node to be influenced by the other nodes in the network and the variability of the context-awareness. As a result, the network could oscillate between several configurations or it could converge to a fixed one. From this new social perspective of multiple criteria decision making, the behaviour of each node is represented by different parameters whose variation determines the dynamics of the social network. The proposed model could be applied to various socio-economic contexts, such as fashion economy, housing location and viral marketing.
\end{abstract}

Keywords: Social network, Context-awareness, Multiple criteria decision making

\section{Background}

In order to extract knowledge from many heterogeneous sources and to study the phenomena within a social network, it becomes fundamental to analyse in detail each process that takes place within it. Indeed, every process that involves a certain number of entities, cannot be analysed only in a macroscopic way, because each single entity contributes to establish the path to follow for the whole system. Looking much more in depth, due to the interactions and the relationships within the network, each entity is influenced in its opinion and, consequently, in its actions (Asavathiratham et al. 2001; Grabisch and Rusinowska 2010a, b; Barjis et al. 2011; Pachidi et al. 2014). As reported in López-Pintado (2008), individual decisions are often influenced by the decisions of other individuals. Without considering any interaction, nodes easily rank criteria in terms of importance following an individual cognitive model (Korhonen and Wallenius 1997). However, every individual cannot be considered as an isolated entity deliberating carefully considered decision. Instead the behavior of each entity is the result of

(c) 2016 Giacchi et al. This article is distributed under the terms of the Creative Commons Attribution 4.0 International License (http://creativecommons.org/licenses/by/4.0/), which permits unrestricted use, distribution, and reproduction in any medium, provided you give appropriate credit to the original author(s) and the source, provide a link to the Creative Commons license, and indicate if changes were made. 
the interaction between its preferences and the dynamic social effects that affect every individual decision (Pentland 2014). These processes of influence are present in different social phenomena such as diffusion of innovations, cultural fads, local variability in crime activities and other conventions that share the contagion logic. As a consequence of the influence exercised by the nodes in the network, the preferences of each node can change during the decision making process bringing therefore to different decisions at different time instants. Focusing on the psychological, social and behavioral aspects of a decision making problem, as done in the Behavioural Operational Research (BOR), would help in making a better use of operational research models (Hämäläinen et al. 2013). A first input to this research field has been made in Korhonen et al. (1990), where an interactive multiple criteria decision making method, paying much more attention to the behavioural realities of decision making, has been proposed. This is why many subjects such as economics, finance and game theory have addressed their attention to the behavioural research topics (Ackert and Deaves 2009; Camerer et al. 2003).

A fundamental aspect in each decision making process is represented by the context in which the decision has to be taken. Different definitions of context have been given in the literature (Liu et al. 2011). However, we think that for the description of our model, the most suitable is "any information that can be used to characterise the situation of an entity" (Abowd et al. 1999). As a consequence, the decisions taken from each node are not dependent on its preferences only but also on the context in which the decisions have to be taken and, more specifically, on the context-awareness. Indeed, a different awareness of the context in which the decision making process takes place can bring to different decisions. For this reason, a model in which the single node is able to take into account the context and, in particular, its variability, is presented in this paper.

In this perspective, to take into consideration all the aspects that characterise a decision making process, not from a static but from a dynamic point of view, in this paper we propose a multiple criteria decision making model being able to represent the dynamics of decisions taken by a node within a social network.

We consider a decision making problem in which a node has to decide among different alternatives evaluated on the basis of several evaluation criteria. The evaluation criteria represent different points of view taken into account by the Decision Maker (DM) to highlight the advantages and the drawbacks of each single alternative (Figueira et al. 2005). The model is based on a weighted sum in which a weight is assigned to each evaluation criterion. The weight represents the importance assigned to the criterion by the node.

The two novelties of the model are the following:

- The variability of the weights of criteria depending on the context in which the decision has to be taken,

- The variability of the context during the time.

On one hand, we assume that the preferences of each node in the network and, consequently, the weights assigned to the different criteria are not fixed during the decision making process. The preferences of the single nodes will evolve depending on their inclination to be more or less influenced by the other components of the network. In 
particular, the preferences will change according to the similarity between nodes. The more the preferences, expressed in terms of weights and past choices, are similar between nodes $n_{h}$ and $n_{k}$, the more the nodes will influence each other.

On the other hand, we assume that the decisions taken by the nodes in the network at previous instants can influence the decision of each node at the current time. Indeed, the consideration of the decisions taken previously by the components of the network brings the node to be more or less aware of the context in which the decision has to be taken.

We shall point out that the inclination of each node to be influenced by the other components of the network, as well as the consideration of the context, causes different dynamics of the decisions taken by the nodes showing that the two different aspects are therefore really relevant in a decision making process.

We think that the proposed model could be applied to different network structures. For this reason, we applied our model to the celebrated El Farol bar problem (Arthur 1994) supposing that the network follows, on one hand, the Erdös-Rényi model (Erdös and Rényi 1959) and, on the other hand, the Barabási-Albert model (Barabási and Albert 1999). In the following, for the sake of simplicity, we will use ER model and BA model instead of Erdös-Rényi model and Barabási-Albert model.

\section{Literature review}

\section{Social networking for decision-making}

Several research works have analysed the importance of social networking in the decision making process. Some of these underline the problem of influence inside networks. In particular, recently some scientists have considered the influence maximisation in viral marketing applications, in which competing entities try to expand their market and maximize their share (Kempe et al. 2015). Anagnostopoulos et al. (2015) present a model for the diffusion of competing alternatives in a social network, in which nodes decide between different alternatives. Nodes usually interact and influence each other, furthermore this influence is not only restricted to the connected neighbours, but also includes those nodes affected by their behaviours, due to social connectedness and contagion (Christakis and Fowler 2013). Social network analysis allows to describe and analyse the interconnections among individuals and how these relationships drive the processes and phenomena inside the network. Therefore, it represents a central analytical tool for understanding the dynamics and diffusion of social behaviours. It allows to unveil how highly connected systems and entities, which form a complex social structure, operate (Aggarwal 2011). In terms of network theory, nodes represent the individual actors, while ties, referred also as edges, links, or connections, are the relationships among individuals. The resulting structures could be different complex graphs. For this reason, graph theory could be applied to describe structurally the relationships between nodes using metrics, such as betweenness, centrality, degree, closeness, clustering coefficient, community detection, etc (Fortunato 2010; Wasserman and Faust 1994). The power of social network analysis is that it produces a different view, where the attributes of individuals are less important than their relationships and ties with other actors within the network. Furthermore, the behavioural dimension means that the individual's actions have to be evaluated not in isolation, but considering the connections with the other players, who can use different strategies (Easley and Kleinberg 2010). All 
these structural and behavioural aspects have to cope with the network dynamics, so that connections and behaviours between nodes change over the time. Large amount of data is available for the case of online social networks. Thus these networks have become much more robust in terms of statistical significance and useful for the verification of some structural properties, such as the small world phenomenon (Watts and Strogatz 1998), preferential attachment (Barabási and Albert 1999), and other structural dynamics. From the decision-making perspective, these relationships, together with the structural properties of the network, could affect the node's decisions also more than the features of the single individual, when considered isolated. Some research studies shed light on the difference between the social mechanisms represented by social selection or homophily [similarity breeds connection (McPherson et al. 2001; Di Stefano et al. 2015)], and influence [the tendency for characteristics and behaviours to spread through social ties such that friends increasingly resemble one another over time, and this influence may affect the choices (Lewis et al. 2012)]. Recent empirical analysis with social network data has suggested that social influence plays an important role in the spread of some behaviours and psychological states (Cacioppo et al. 2009; Christakis and Fowler 2007, 2008). In fact, Christakis and Fowler have suggested how social influence is significant in some phenomena, such as the spread of obesity, smoking, or happiness. These behaviours spread through the network, producing a social contagion effect. More than social influence, this social contagion process is able to amplify the spread of information in a social network, and this is the reason why understanding the mechanics of social contagion is crucial to predict how far it will spread and with what intensity. In terms of decision making, social influence mechanisms have been analysed in several works. In Hoede and Bakker (1982), an acceptance-rejection decision-making problem, in which each node in a social network has to choose between being in favor or against a certain decision, is taken into account. The basic assumption is that each node has its own inclination towards a certain decision and its final choice can be different from this inclination, due to the influence of the other nodes in the network. Without taking into account any external cause of influence, the final choice of the node can be different from its inclination only due to the influence exercised from the other nodes in the network. The social influence mechanism can also be described, taking into account the example reported in Rusinowska and de Swart (2006). The authors use a vector to represent the inclination of each node; different values imply different degrees of influence among nodes. In Grabisch and Rusinowska (2010a), a direct and an opposite influence are defined. On one hand, the direct influence is ruled from a coalition when it succeeds in leading the decision of a single node to the coalition's inclination, even if the node's inclination was different. On the other hand, a coalition exercises an opposite influence on a single node when, although they have the same inclination, the node decides in a different way. In contrast with Hoede and Bakker (1982) and Grabisch and Rusinowska (2010a), in Grabisch and Rusinowska (2010b), the node has an ordered set of possible actions to choose from, and the concepts of direct and opposite influences are generalised using the concepts of positive and negative influences. The positive influence measures the attraction carried on by a coalition on a node having at the beginning an inclination different from that one of the coalition while, eventually, the final choice is closer to the coalition's inclination. The negative influence is instead exercised in the 
opposite direction, but also in this case the final decision is a consequence of the coalition's influence. The mechanism of social influence is also considered in the model proposed in Asavathiratham et al. (2001) for large and complex engineered networks, like power grids, communication networks, etc. In the model, the authors try to understand the basic features of the network's global behaviour and the reason of certain spatial and temporal organisation. Based on a network of interacting Markov chains, where each chain corresponds to a node of the network and it is admitted that each chain can differ from one site to another, one of the most relevant aspects is the influence of the other chains on the dynamics of each one related to its neighboring sites on the network. In the same direction, López-Pintado (2008) presents a dynamic model in which the choice of a node to adopt or not a particular behaviour is a function of the actions made by its neighbors, which are a random sample of the total population in the previous step.

\section{Context and context-aware applications}

The consideration of context inside social networks has grown during the last decades and several attempts to formalize its definition have been made in literature. Schilit et al. (1994) stated that context is linked to the location, nearby person, host or objects, and their evolution over time. Even if in the scientific literature are present many and different definitions of context (Brown et al. 1997; Liu et al. 2011; Snowdon and Grasso 2000; Ahn and Kim 2006), there is not a standard definition of context. Several researchers accept the definition given in Abowd et al. (1999), where context is defined as any information that can be used to characterise the situation of an entity. An entity is a person, place or object that is considered relevant to the interaction between a user and an application, including the user and applications themselves. Consequently, as pointed out in Abowd et al. (1999), a system is context-aware if it uses context to provide relevant information and/or services to the user, where relevancy depends on the users's task. Accordingly, the ability of mobile user's applications to discover and react to changes in the environment they are in, is defined as "context-awareness" and it was firstly introduced in Schilit and Theimer (1994). Taking into consideration the different ways the system reacts to the changing conditions of the environment, context-awareness has been classified in Chen et al. (2000) as follows:

- Active context-awareness: the system adapts itself to the changing environment modifying its behaviour,

- Passive context-awareness: the system presents the new or updated context to an interested user without modifying its behaviour.

Context-aware applications have several fields of application, like healthcare and wellbeing, transportation and location, social networking and environmental monitoring. This has been made possible through the integration of context ubiquitous sensing, micro-sensors and the geographic information systems (GIS). For example, regarding the healthcare field, several E-health applications have been proposed to monitor patients with different diseases, in order to guarantee an adequate level of assistance. Particularly in these cases, it is important to choose necessary context information in order to extract useful information and obtain knowledge that can permit to adapt dynamically the behaviour of the system according to the environment characteristics (Guermah et al. 2013). Considering, instead, the social networking, the SAMOA (Socially Aware and Mobile Architecture) 
framework (Bottazzi et al. 2007), allows mobile users to create social networks, following its movements. It is based on two kinds of context visibility: place visibility (place awareness) and profile visibility (profile awareness).

\section{A unified framework of social influence and context-awareness}

In the decision making model presented in Giacchi et al. (2014), each process taking place in a social network is characterised by two main features: complexity and dynamism. To perform the different processes, each node has to take into account not only its personal knowledge but also the influences perceived from its surroundings. Considering this scenario, every decision will be the result of a complex and dynamical process, affecting the present and the future status of the node.

Consequently, given a set of alternatives $A$ and a set of criteria $G$ within social network, the definition of decision provided in Wang and Ruhe (2007)

$$
d=f(A, G)=f: A \times G \rightarrow A
$$

has to be extended including the influence that a node can have on the preferences of the other nodes in the network. In this context, it is important to distinguish between positive and negative influences. On one hand, a node $x$ positively influences a node $y$, if $x$ supports $y$ in its decisions while, on the other hand, $x$ negatively influences $y$, if $x$ acts leading $y$ to wrong decisions. For this reason, before making its decisions, the node has to become aware of what it has nearby and what resources it has. A crucial part of the decision process is therefore the context-awareness, that allows a node to make an aware cognitive decision on the basis of the available information.

Furthermore, in Giacchi et al. (2014) it has been taken into account a particular scheme of the decision's criteria that, as a result of the interaction among nodes in the network, can assume different priorities depending on three dimensions: Time, Context and Awareness. For example, in a context $K_{1}$ and at time $t_{1}$, as a consequence of the interactions among the nodes in the network and through processes of adaptation (Cioffi-Revilla 2013) and social contagion (Christakis and Fowler 2007), criterion $g^{i_{1}}$ may be perceived as the most important one, while in another context $K_{2}$ and at a different time $t_{2}$, criterion $g^{i_{2}}$ may be considered as the most important one.

So, applying this assumptions, the inference process not only has a dependence from given and from memory as indicated in Gigerenzer and Goldstein (1996), but also from social relationships, determined by the network properties and structure and by the perceived influences.

\section{Brief introduction to MCDM}

In a Multiple Criteria Decision Making (MCDM) problem [see Figueira et al. (2005) for a collection of surveys on MCDM], a set of alternatives/actions $A=\{a, b, \ldots\}$ is evaluated with respect to a finite and coherent family of criteria $G=\left\{g^{1}, g^{2}, \ldots, g^{p}\right\}$ (Roy 1996), that is exhaustive (all relevant criteria are taken into account), cohesive (if $a$ is at least as good as $b$ for all but one criteria and $a$ is better than $b$ on the remaining criterion, then $a$ should be preferred to $b$ ) and non-redundant (removing one criterion from the family renders it not exhaustive or cohesive). We suppose that each criterion is a real valued function $g^{i}: A \rightarrow \mathbb{R}$ having an increasing direction of preference (the higher the 
evaluation of $a$ on criterion $g^{i}$, the better $a$ is) or a decreasing direction of preference (the higher the evaluation of $a$ on criterion $g^{i}$, the worse $a$ is). For example, in a project evaluation problem, different projects (the alternatives in our terminology) can be evaluated with respect to different aspects such as, Opportunity, Potential Risks, Technology, Finance and Employment (Tavana et al. 2015). Investment cost and Return on Investment can be considered subcriteria of the financial aspect, while Impact and Technology Importance can be highlighted as subcriteria in the Technology aspect. Investment Cost has a decreasing direction of preference, while Return on Investment, Impact, and Technology Importance have an increasing direction of preference.

Three main problems are considered in MCDM: choice, ranking and sorting. Choice problems consist into choosing one or more alternatives (actions) considered the best; ranking problems consist into rank ordering all alternatives from the best to the worst, while sorting problems consist into assigning each alternative to one or more contiguous classes preferentially ordered from the DM. In the considered example, the DM can be interested in choosing the best project, in ranking all of them, or in assigning to classes, such as "bad", "medium", "good", ordered with respect to their reliability.

Looking at the evaluations of the alternatives on the considered criteria, the only information that can be gathered is the dominance relation ${ }^{1}$ but, especially in case of a great number of criteria, this relation is really poor since, very often, comparing alternatives $a$ and $b, a$ is preferred to $b$ on some criteria while $b$ is preferred to $a$ on the remaining ones. For this reason, one needs to aggregate the evaluations of the alternatives to get some recommendations with respect to the problem at hand. Three different ways of aggregating the evaluations are the most known in MCDM:

- assigning a real number to each alternative being representative of its degree of desirability as in the Multiple Attribute Value Theory (MAVT) (Keeney and Raiffa 1993);

- comparing alternatives pairwise by means of binary relations, to check if one is at least as good as the other or viceversa as in the outranking methods (Brans and Vincke 1985; Figueira et al. 2013);

- using a set of "if...then" decision rules as in the Dominance Based Rough Set (DRSA) approach that, starting from preferences provided by the DM, induces some rules expressed in a natural language (Greco et al. 2001).

The first two families of aggregation methods are based on some parameters such as weights of criteria, marginal value functions, indifference, preference and veto thresholds, etc. that can be obtained in a direct or in an indirect way. In the first case, the DM is able to provide directly values to all of these parameters, while in the second one the DM provides some preference on reference alternatives, from which parameters compatible with these preferences can be elicited. Since the direct preference information involves a great cognitive effort from the part of the DM, the indirect technique is the most used in practice (Jacquet-Lagrèze and Siskos 2001). In the indirect way of providing preference information and calling compatible model a set of value parameters restoring the preferences provided by the DM, more than one model could be compatible with them. Each of these models provides the same recommendations on the reference alternatives but

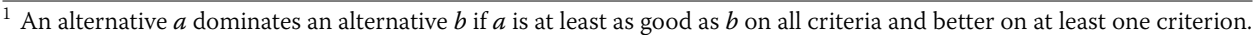


different recommendations on the other alternatives on which the DM did not provide any preference information. Since, using only one compatible model can be considered arbitrary to some extent, Robust Ordinal Regression (ROR) (Corrente et al. 2014; Greco et al. 2008) takes into account simultaneously all models compatible with the preferences provided by the DM building a necessary and a possible preference relation. The necessary and possible preference relations hold between two alternatives $a$ and $b$ if $a$ is at least as good as $b$ for all or for at least one compatible model, respectively.

\section{A dynamic multiple criteria decision making model with a social perspective}

As previously described, a social decision making process is characterised by two fundamental aspects, that are, the dynamism and the context-awareness.

We shall suppose that $m$ nodes are individually involved in a decision making choice problem in which a finite set of alternatives is evaluated with respect to $p$ criteria.

In the description of our model we shall use the following notation:

- $N=\left\{n_{1}, \ldots, n_{h}, \ldots, n_{m}\right\}$, a finite set of nodes;

- $A=\{a, b, \ldots\}$, a finite set of alternatives;

- $G=\left\{g^{1}, \ldots \ldots, g^{p}\right\}$, a finite set of criteria.

Since each decision depends on the context in which it has to be taken and, as defined in Abowd et al. (1999), context is dependent on the information at hand that varies over time, the variability of the context should be taken into account in each decision problem. For this reason, in our model we consider a further criterion $g^{p+1}$, such that $g^{p+1}(a ; t)$ is the evaluation of $a$ relative to the considered context at time $t$. This evaluation is not fixed over time but it varies according to the variability of the context, following a rule that we shall describe later. Note that the introduction of criterion $g^{p+1}$ implies that the new set of criteria that has to be considered in the decision problem is $G=\left\{g^{1}, \ldots \ldots, g^{p}, g^{p+1}\right\}$.

The preferences of node $n_{h}$ are represented by the vector of weights $w_{h}=\left(w_{h}^{1}, \ldots, w_{h}^{p}, w_{h}^{p+1}\right)$, where $w_{h}^{i}$ is the importance given to criterion $g^{i}$ by node $n_{h}$. As can be noticed, we introduced also the weight $w_{h}^{p+1}$ of criterion $g^{p+1}$, which represents the importance given by node $n_{h}$ to the variability of the context and being dependent on its context-awareness.

Definition 1 Given a node $n_{h}$, its vector of weights $w_{h}=\left(w_{h}^{1}, \ldots, w_{h}^{p}, w_{h}^{p+1}\right)$ and the vector $g(a ; t)=\left(g^{1}(a), \ldots, g^{i}(a), \ldots, g^{p}(a), g^{p+1}(a ; t)\right)$ composed of the evaluations of alternative $a \in A$ at time $t$, the comprehensive value of $a$ is obtained as follows:

$$
U_{h}(a ; t)=\sum_{i=1}^{p}\left[w_{h}^{i} \cdot g^{i}(a)\right]+w_{h}^{p+1} \cdot e^{-g^{p+1}(a ; t)} .
$$

On the basis of Eq. (2), we shall consider the preference relation $\succsim_{h}^{t}$ of node $n_{h}$ at time $t$ defined as follows:

$$
a \succsim_{h}^{t} b \quad \text { iff } \quad U_{h}(a ; t) \geq U_{h}(b ; t), \quad a, b \in A .
$$


Consequently, node $n_{h}$ will choose the alternative $a \in A$ such that $a \succsim_{h}^{t} b$ for all $b \in A$, that is $U_{h}(a ; t)=\max _{b \in A} U_{h}(b ; t)$. Without loss of generality, in Eq. (2) we shall suppose that criteria $g^{1}, \ldots, g^{p}$ have an increasing direction of preference while criterion $g^{p+1}$ has a decreasing direction of preference. In the following, we shall provide two examples to explain the meaning of the variability of the context and to justify the decreasing direction of preference of criterion $g^{p+1}$.

Example 1 Suppose that a consumer has to buy a good, choosing it among a set of alternative goods. These goods are evaluated with respect to different criteria, such as quality, aesthetics and price. While the evaluation of the goods with respect to quality and aesthetics can be supposed constant over time, the price of the goods evolves in consequence of the choices made by the other DMs in the considered market. Such a variability of the price can be included in our model as the variability of the context in which the choice has to be made. Obviously, in this problem price will have a decreasing direction of preference.

Example 2 Being inspired by the El Farol bar problem (Arthur 1994), suppose that a consumer has to choose a bar to spend the evening. The bars are evaluated on the basis of criteria such as location, quality of service and people attendance. Moreover, suppose that DMs prefer less crowded bar. While the location of the bars and the quality of the provided service can be supposed not variable, the frequency of people going in the bars changes over time in consequence of the choices made by the other DMs. In this case, the variability of frequency of people in each bar can be interpreted as the variability of the context. Furthermore, the preference of the DM for less crowded bars justifies the decreasing direction of preference of criterion $g^{p+1}$.

Note 1 Let us observe that in the two examples we provided above, a great number of nodes choosing an alternative a will affect negatively the comprehensive evaluation of a. In the first example, the increase of the price is obviously not appreciated by the buyer and, analogously, in the second example, the increase of the number of people going in the bar will reduce the appreciation of the customer for the same bar.

In some other cases, the increase of the number of nodes choosing an alternative a will affect positively the comprehensive evaluation of a. For example, in the fashion market, the increase of the number of people choosing a good will increase the appeal of the same good generating, therefore, an increase of the good demand and an imitation effect in the other buyers. In these cases, Eq. (2) should be modified replacing $w_{h}^{p+1} e^{-g^{p+1}(a ; t)}$ with $w_{h}^{p+1} \cdot\left(1-e^{-g^{p+1}(a ; t)}\right)$ so that the comprehensive evaluation of alternative a will increase due to the fact that several nodes have chosen this alternative. These cases have been studied in the models of herd behavior (Avery and Zemsky 1998; Banerjee 1992; Bikhchandani et al. 1992; Brunnermeier 2001). Of course we can have cases in which both an attractive and a repulsive effect of increasing the number of customers can be simultaneously present.

Being the node $n_{h}$ part of a network, its preferences can change during the decision making process as a consequence of the influence that the nodes in the network can exercise on it and on the node's inclination to be affected by these influences. On 
one hand, a node that is not influenced at all by any other node in the network will not change its preferences. On the other hand, a node more or less influenced by the other nodes will modify its preferences taking more into account the preferences of the nodes closer to it and the preferences of the nodes that have made similar decisions in the past. As a consequence of the previous remarks, the weight $w_{h}^{i}(t)$ assigned to criterion $g^{i}$ by node $n_{h}$ at time $t$ will change according to the following law:

$$
w_{h}^{i}(t)=\delta_{h} w_{h}^{i}(t-1)+\left(1-\delta_{h}\right) \frac{\sum_{k \neq h} w_{k}^{i}(t-1) \cdot f\left(d_{h k}(t-1)\right) \cdot a_{h k}}{\sum_{k \neq h} f\left(d_{h k}(t-1)\right) \cdot a_{h k}}
$$

where:

- $a_{h k} \in\{0,1\}$ is an element of the adjacency matrix $A_{h k}$ representing the considered network; if $a_{h k}=0$, then nodes $n_{h}$ and $n_{k}$ are not linked and do not influence each other while, if $a_{h k}=1$, then nodes $n_{h}$ and $n_{k}$ are linked and they can influence each other. For the sake of simplicity, we shall suppose that $A_{h k}$ is symmetric and therefore the influence exercised by $n_{h}$ over $n_{k}$ is the same as the influence exercised by $n_{k}$ over $n_{h}$. Consequently, for each node $n_{h}$ we can define the set $N_{h}=\left\{n_{k} \in N: a_{h k}=1\right\}$, that is the set of nodes that are linked to $n_{h}$ and that could influence its decisions.

- $\delta_{h} \in[0,1]$ represents the node's inclination to be influenced by the nodes belonging to $N_{h}$; the less $\delta_{h}$, the more nodes in $N_{h}$ will influence the preferences of $n_{h}$; in particular, if $\delta_{h}=0$, then the preferences of $n_{h}$ will be completely dependent on the preferences of the nodes in $N_{h}$, while, in the opposite case, if $\delta_{h}=1$, then the preferences of $n_{h}$ are not affected by the nodes in $N_{h}$. For the sake of simplicity we shall suppose that $n_{h}$ can be influenced in the same way by nodes in $N_{h}$, however, it could be reasonable to consider an inclination $\delta_{h k}$, representing the inclination of $n_{h}$ to be influenced by $n_{k}$;

- $f\left(d_{h k}(t-1)\right)$ is the importance given by $n_{h}$ to the preference of node $n_{k}$ on criterion $g^{i}$ at time $t-1\left(w_{k}^{i}(t-1)\right)$; moreover $f$ is a non-decreasing function of the distance $d_{h k}(t-1)$ that will be described later. For the moment, we assume that:

$$
f\left(d_{h k}(t-1)\right)=\frac{1}{d_{h k}^{2}(t-1)} .
$$

The idea under Eq. (3) is that the preferences' dynamics of node $n_{h}$ is dependent on its inclination to be influenced by nodes belonging to $N_{h}$. In particular, on one hand, preferences of $n_{h}$ at time $t$ will be dependent on its preferences at time $t-1$ and, on the other hand, $n_{h}$ will give a weight to the preferences of node $n_{k} \in N_{h}$ depending on the distance $d_{h k}(t-1)$ between the two nodes. This distance is computed in terms of similarity between the preferences of the two nodes and in terms of similarity between the choices made by the two nodes at previous times. The idea is that the more the preferences (the weights and the past choices) are similar between nodes $n_{h}$ and $n_{k}$, the more $n_{k}$ influences node $n_{h}$.

Obviously, the more similar are preferences and choices of nodes $n_{h}$ and $n_{k}$, the more importance will be assigned to the preferences of $n_{k}$ from $n_{h}$. 
From an analytical point of view, $d_{h k}(t)$, that is the distance between nodes $n_{h}$ and $n_{k}$ at time $t$, is defined in the following way:

$$
d_{h k}(t)=\sqrt{\sum_{i=1}^{p}\left[w_{h}^{i}(t-1)-w_{k}^{i}(t-1)\right]^{2}}+x_{h k}(t)
$$

The first part of $d_{h k}(t)$ is the Euclidean distance between the weights vectors of $n_{h}$ and $n_{k}$ representing the distance between the preferences of the two nodes. The second part, instead, is a measure of the number of times nodes $n_{h}$ and $n_{k}$ have taken different decisions in the previous considered time instants. With respect to the second part, the importance given to the decisions will be dependent on the instants in which they have been taken. In particular, the more recent they are, the more importance they have. Formally, $x_{h k}(t)$ can be expressed as:

$$
x_{h k}(t)=\sum_{r=1}^{\# P T} \beta \gamma^{r-1}
$$

where:

- \#PT is the number of considered previous time instants and it can be interpreted as the memory of the system. If \#PT $=0$ then the system will be memory less. Therefore the output of the system will be based only on the current system state and it will not take into account its history. Instead, if the system has a memory, as proposed in our model, the output of the system is not dependent on the current state only but also on some previous instants. This can be considered an important property of the system, because having memory of what previously happened, influences the behaviour of each single node, contributing to increase or decrease the distance between nodes, as indicated in Eq. (5).

- $\beta= \begin{cases}1 & \begin{array}{l}\text { if at the considered time instant, nodes } n_{h} \text { e } n_{k} \\ \text { have not taken the same decision } \\ \text { otherwise }\end{array}\end{cases}$

- $\gamma \in[0,1]$ is a damping coefficient used to weigh the decisions taken in different time instants.

Let us observe that the two parts of Eq. (5) are not expressed in the same scale. Indeed, the distance between the weight vectors can assume values in the interval $[0, \sqrt{2}]$, while $x_{h k}(t)$ can take a value in the set $\left\{0, \gamma, \ldots, \gamma^{\# P T-1}\right\}$. To make commensurable these two values, we normalize $x_{h k}(t)$ using the following equation:

$$
x_{h k}(t) \cdot \frac{\sqrt{2}(1-\gamma)}{1-\gamma^{\# P T}} .
$$

In this way $x_{h k}(t)$ will assume values in the interval $[0, \sqrt{2}]$ as the first part of Eq. (5).

As explained above, the variability of the context plays a central role in the dynamics of node's preferences. Since the modification of the context is due to the decisions taken by all nodes in the network, we assume that the evaluation of an alternative at time $t$ and, 
therefore, in a certain context, depends on its evaluation at time $t-1$ and on the number of times the alternative has been chosen by the nodes in the network at times $t-1$ and $t-2$. Formally, the evaluation of alternative $a$ at time $t$ in a considered context will be obtained as follows:

$$
g^{p+1}(a ; t)=g^{p+1}(a ; t-1)+\alpha \frac{[M(a ; t-1)-M(a ; t-2)]}{|N|}
$$

where $M(a ; t-1)$ and $M(a ; t-2)$ indicate the number of times that the alternative $a$ has been chosen at the time instants $t-1$ and $t-2$, while $\alpha$ is a coefficient that represents the relevance of the increase or decrease of the number of times that alternative $a$ has been chosen in the variation of the context.

Referring to Examples 1 and 2, in the first one the variation of the context will provide a variation on the price of the considered goods while, in the second one, the variation of the context will affect the number of people going into the bar. On one hand, the increase of the demand of a particular good will cause an increase of its price while, on the other hand, the increase of the number of people going into a bar will convince more other people not to go there.

\section{Main components of the model}

For a given network, i.e. for given values $a_{h k}$, the two components that affect the dynamics of the model are $\delta_{h}$ and $\alpha$.

As introduced in the section above, $\delta_{h}, h=1, \ldots, m$, represents the node's inclination to be influenced by the other nodes in the network connected to it. So, its introduction, as a parameter that influences the dynamics of the entire system, is representative of the behavioural sphere, and in particular of the node volition to be part of a community and to take into account the others' opinion. $\delta_{h}$ can assume values in the interval $[0,1]$. If $\delta_{h}=0$, then node $n_{h}$ is totally influenced by other nodes in the network to which it is connected. Notice that, in Eq. (3) the first term will be equal to 0, and the weights updating process will continue till the new weight will be equal to the barycenter of the group. Instead if $\delta_{h}=1$, then $n_{h}$ will not take into account the others' opinion and its preferences will not change. Unlike the previous case $\left(\delta_{h}=0\right)$, and according to Schelling (1969), this can be considered a point of stable equilibrium, where a mechanism of complete segregation is active and no node will move towards another choice.

In addition to $\delta_{b}$ another important parameter of the model is $\alpha$. It is a coefficient that represents the relevance of the increase or decrease of the number of times that an alternative has been chosen in the variation of the context. The introduction of $\alpha$ changes the behaviour of the nodes, inducing the whole system to oscillate much more before stabilizing itself. As pointed out in the literature review section, context plays a central role because different issues can have different perception and representation, due to different contexts, leading to different level of awareness about the task. The parameter $\alpha$ can assume values greater than zero. If $\alpha$ is equal to zero, the context has no importance on the system's dynamics, because it assumes always the same value, and it can be compared to a static model. 


\section{Model performance and discussion}

In this section, we shall apply the proposed model to Example 2 considering two network structures. In the first part of the section, we assume that the network follows the ER model (Erdös and Rényi 1959). We highlight how the variation of the main components of our model, that are the nodes' inclination and the variability of the context, will affect the dynamics of the preferences.

In the second part, instead, we assume that the network follows the BA model (Barabási and Albert 1999) and we underline how the dynamics of preferences is subject to the network structure modification.

As shown in Fig. 1, in the ER model, all nodes have approximately the same number of connections, while in the BA model only some nodes have high degree. For example, considering node $n_{13}$ in Fig. 1 , one can see that in the ER model, shown in the left side, its degree is 16 , while in the BA model, shown in the right side, its degree is 1.

Our network is composed of $m=100$ nodes representing customers that have to choose a bar to spend the night. Each bar is evaluated on three criteria Location (L), Quality of Service (QoS) and People Attendance (PA) as shown in Table 1. We suppose that the evaluations of the four bars with respect to $L$ and QoS are expressed on a $[0,1]$ scale and both of them have an increasing direction of preference. Since PA expresses the variability of the context in which nodes have to make their choices, the evaluations of the bars on this criterion are variable over time, while evaluations on L and QoS are supposed fixed. In particular, we assume that the evaluations of the four bars with

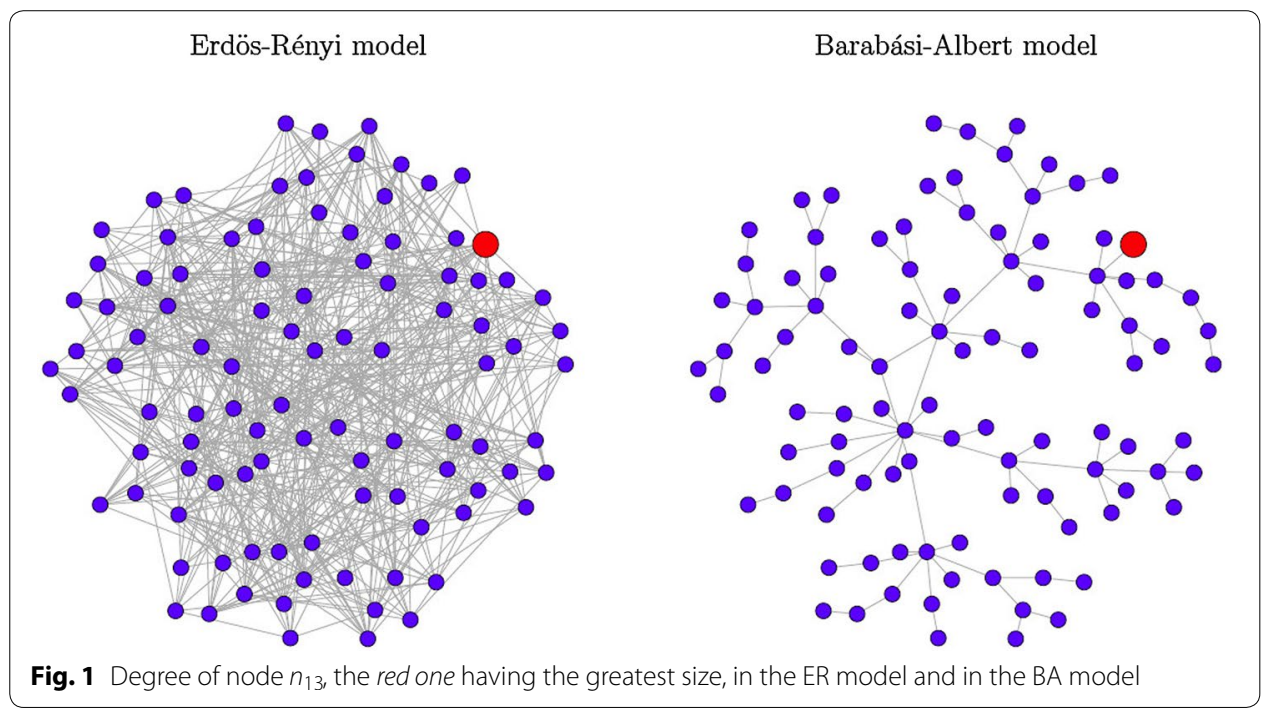

Table 1 Evaluations of the bars on the three considered criteria

\begin{tabular}{llll}
\hline Bar/criteria & Location (L) & Quality of service (QoS) & People attendance (PA) \\
\hline bar $_{1}$ & 0.684 & 0.086 & 0.058 \\
bar $_{2}$ & 0.452 & 0.682 & 0.192 \\
bar $_{3}$ & 0.259 & 0.851 & 0.177 \\
bar $_{4}$ & 0.203 & 0.891 & 0.400 \\
\hline
\end{tabular}


respect to PA presented in Table 1 are based on an estimate of the frequency of the customers in the considered bars. To each node a vector of weights is associated and each of them represents the importance given by the node to the corresponding criterion.

We consider a network configuration with probability $p=0.1$, where $p=0.1$ is the probability of having a connection between two nodes. The network will be represented by a graph whose vertices are the nodes of the network. Each node will be colored according to the choice made. In all Figures in this section, the colors associated to the four alternatives are those shown in Table 2.

To study the behaviour of the model in a simulation environment, we assume that:

- the inclination of each node $n_{h}$ to be influenced by the other nodes in the network is represented by a value $\delta_{h}$ taken uniformly in the interval [0,1];

- $\alpha=1$

- there is not any external cause of influence and, therefore, the preference of the nodes will change only as the effect of the influence exercised by the other network's nodes;

- the network configuration does not change over time in the sense that the nodes in the considered network, as well as their mutual connections, do not change over time;

- starting from time $t_{0}$, in order to update the evaluations of the four bars with respect to the considered context (the evaluations of the alternatives on criterion PA), the choices made by the network's nodes at time $t_{0}-1$ are represented by the vector 26-22-24-28 meaning that, 26 people choose bar $_{1}, 22$ choose bar $_{2}$ and so on. Moreover, the choices made by the nodes at time $t_{0}$ are the consequence of the application of Eq. (2) to the evaluations in Table 1. For example, considering the starting vector of weights $\left(w_{1}^{1}, w_{1}^{2}, w_{1}^{3}\right)=(0.491,0.173,0.336)$ and the bars' global evaluations $(0.668,0.617,0.556,0.479)$, node $n_{1}$ will therefore choose bar ${ }_{1}$.

Note that in all performed simulations, we considered \#PT equal to 17 , since we observed that considering a value greater than 17 does not affect the dynamics of the choices.

The first characteristic we would like to highlight is the dynamic behaviour of the nodes' choices, as shown in Fig. 2, where we reported the configurations of the network with respect to the choices made by its nodes, for the first iterations. As we can see, at time $t_{0}$, the choices made by the nodes in the network are 41-15-37-7 while, as a consequence of the influence mechanism and due to influences on nodes' inclinations, at time $t_{0}+5$ the choices made by the nodes became 3-79-0-18.

The variation in the choices made by the nodes at different iterations is due to three main reasons: the variation of the importance assigned to the different criteria, the

Table 2 Colors associated to the nodes depending on the choice made

\begin{tabular}{ll}
\hline Bar & Color choice \\
\hline bar $_{1}$ & Red \\
bar $_{2}$ & Yellow \\
bar $_{3}$ & Blue \\
bar & Green \\
\hline
\end{tabular}




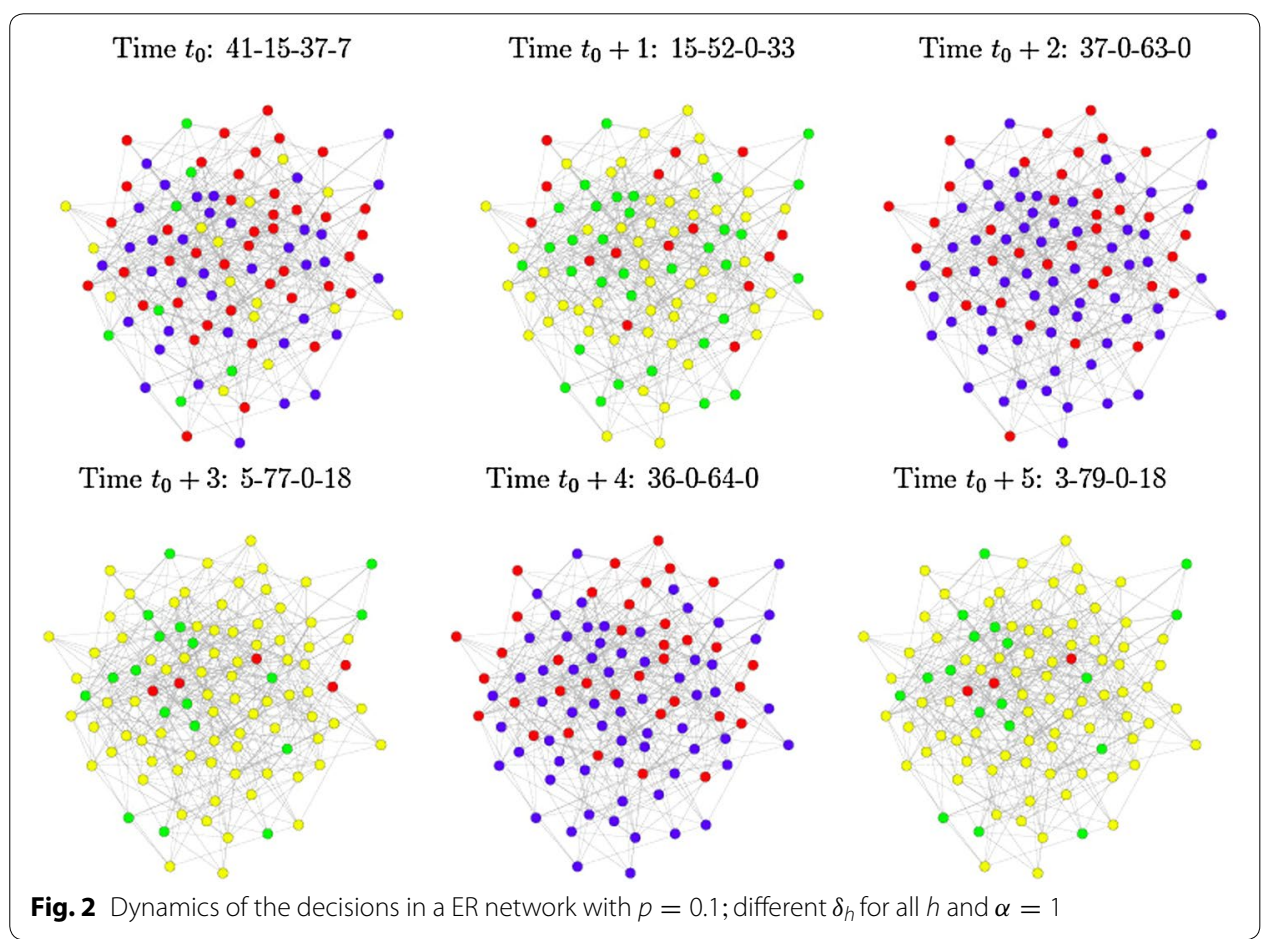

inclination of each node to be influenced by the other nodes to which it is connected in the network, and the variation of the context. Regarding the first point we report, as example, the dynamics of weights of node $n_{30}$ during the first considered iterations, shown in Fig. 3 on the left. As we can see, at the beginning, the most important criterion for this node is L followed by PA and QoS; as a consequence of the influence mechanism, at iteration $t_{0}+9$, criteria $\mathrm{L}$ and QoS assume, more or less, the same importance. Then, at iteration $t_{0}+13$ criterion QoS becomes more important than PA. In the end, QoS is the most important criterion for $n_{30}$ while, at the beginning it was the lowest important one.

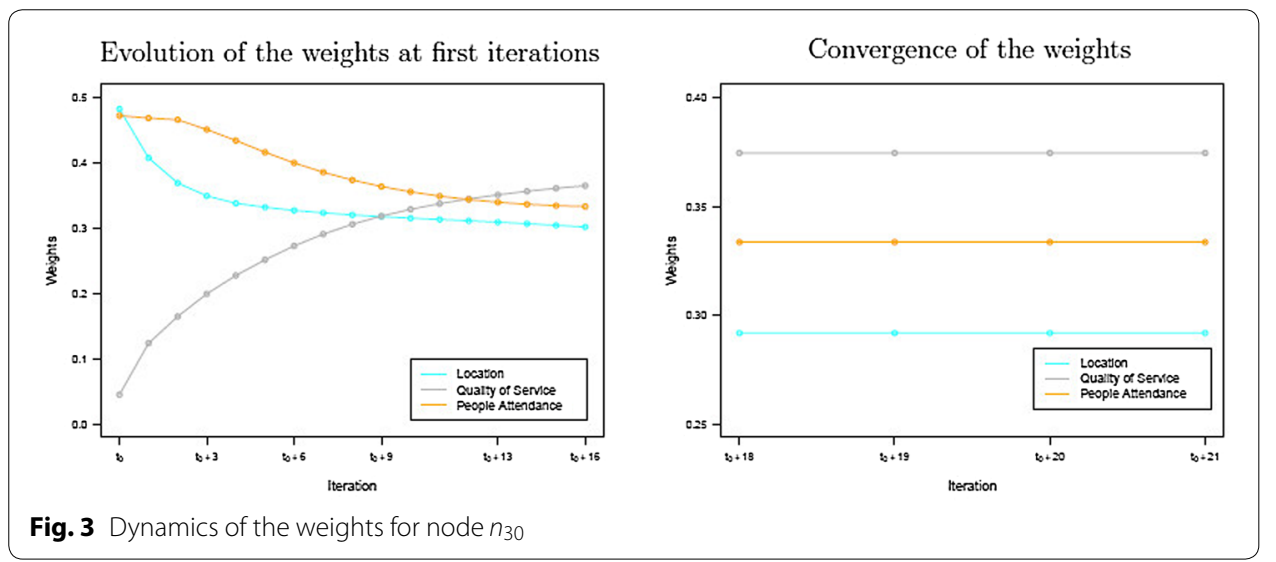


As already mentioned before, applying Eq. (3), iteration after iteration, the preferences of a node will be always closer to the preferences of the nodes that have taken similar decisions in the previous iterations and more different from the preferences of the nodes that have taken different decisions. Therefore, just after a certain number of iterations, a cluster effect will appear in the network so that nodes that have taken similar decisions in the past will have approximately the same preferences and the weights assigned to the different criteria will be subject only to very slight modifications as shown in Fig. 3 on the right.

The second reason for the dynamics of choices is the inclination of each node to be influenced. To highlight this aspect, we compare two networks which differ for the inclination of 10 nodes only, that is, 90 nodes have the same value of $\delta_{h}$ in both cases while $\delta_{h}$ of the 10 remaining nodes are pairwise exchanged. In this way the average inclination $\delta_{h}$ of the nodes in the two networks is the same.

Note 2 Let us underline that when we state that we modified a parameter, we left all the other parameters fixed as listed in the above assumption. For example, in this comparison, we modified $\delta_{h}$ only, while $\alpha=1$ and the vector representing the choices made by the nodes at time $t_{0}-1$ is 26-22-24-28.

As shown in Fig. 4 , only one node $\left(n_{40}\right)$, the node having the greatest size in the Figure, makes a different choice in the two networks, because it chooses the first alternative in the first case, while it chooses the third alternative in the second case. Even if this change could appear not significant, we would like to observe that in the two networks the number of connections and the nodes to which they are connected do not change. Consequently in modifying its preferences, each node will be influenced by the same nodes. The only difference is that 10 out of the 100 nodes change their inclination $\delta_{h}$ to be influenced and, in particular, $n_{40}$ is exactly one of them.

The third aspect, causing the dynamics of the choices and that represents one of the novelty introduced in the paper, is the variability of the context. In particular, here we

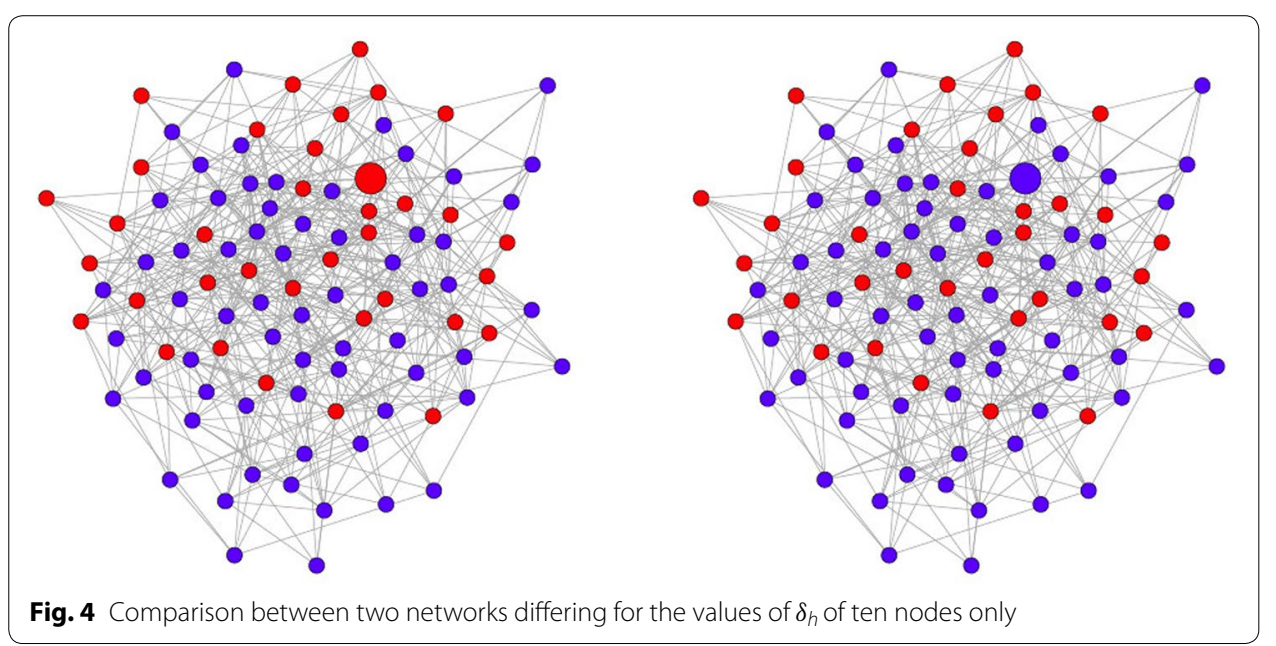


would like to show how different values of $\alpha$ in Eq. (8) affect the dynamics of the preferences. For this reason we compare the dynamics of the same network varying only the value of $\alpha$. In the first network we consider $\alpha=1$, while in the second network, $\alpha$ is equal to 2 . Therefore the variability of the context is greater in the second case.

As we can see in Fig. 5, different values of $\alpha$ have a different impact on the dynamics of the choices made by the nodes in the network since 28 of them make different choices in the two cases.

Let us underline here once more the importance of taking into account the variability of the context in our model. As already observed above, according to the dynamics of weights described by Eq. (3), after a certain number of iterations the weights of the criteria will not change anymore (see Fig. 3 on the right) and this implies that the preferences of the node for the considered criteria will not be subject to any modification. Nevertheless, the introduction of the variability of the context may bring to an oscillation of the decisions taken from the different nodes as observed in Table 3a.

In the first iterations, the configurations of the network will vary more deeply since two phenomena occur simultaneously, that are, the modification of the preferences due to dynamics of the weights described by Eq. (3) and the variability of the context

Configuration of the network for $\alpha=1: 3-79-0-18$ Configuration of the network for $\alpha=2: 2-52-0-46$
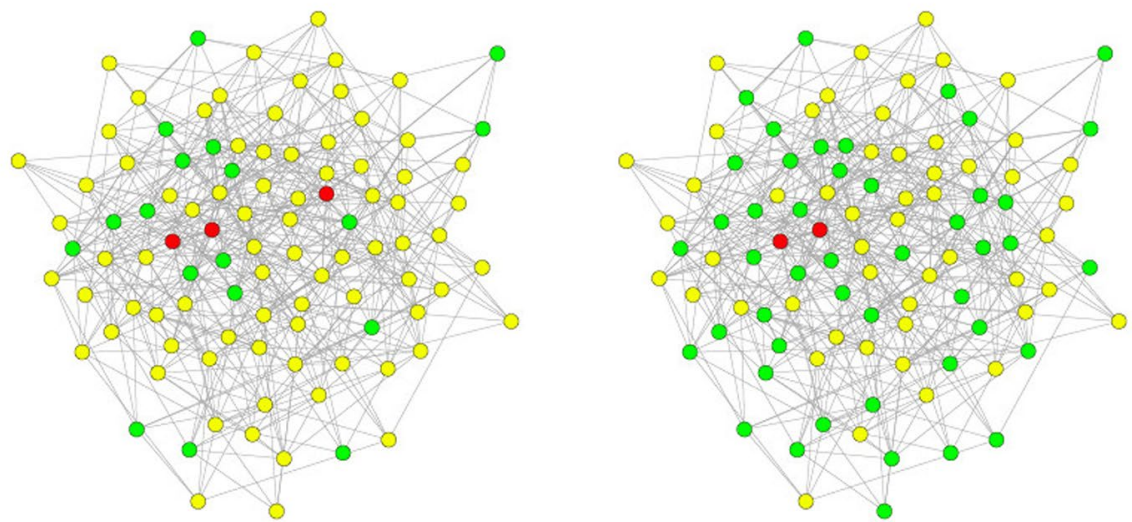

Fig. 5 Comparison between the same network considering different values of $\alpha$ in Eq. (8)

Table 3 Dynamics of the decisions taken by the nodes in the network considering $\alpha=1$ and $\alpha=0$, respectively

\begin{tabular}{llll}
\hline Time & Configuration & Time & Configuration \\
\hline (a) $\alpha=1$ & & (b) $\alpha=0$ & \\
$t_{0}$ & $41-15-37-7$ & $t_{0}$ & $41-15-37-7$ \\
$t_{0}+1$ & $15-52-0-33$ & $t_{0}+1$ & $37-17-44-2$ \\
$t_{0}+2$ & $37-0-63-0$ & $t_{0}+2$ & $37-17-44-2$ \\
$t_{0}+3$ & $5-77-0-18$ & $t_{0}+3$ & $36-18-45-1$ \\
$t_{0}+4$ & $36-0-64-0$ & $t_{0}+4$ & $36-18-46-0$ \\
$t_{0}+5$ & $3-79-0-18$ & $t_{0}+5$ & $36-17-47-0$ \\
$t_{0}+6$ & $36-0-64-0$ & $t_{0}+6$ & $36-17-47-0$ \\
$t_{0}+7$ & $3-79-0-18$ & $t_{0}+7$ & $36-17-47-0$ \\
\hline
\end{tabular}


described by Eq. (8). In Table 3b, one can instead observe a different dynamics of the network due to the value of $\alpha=0$, implying that the variability of the context is not taken into account in the dynamic of the network. At the beginning, the network passes through different configurations in consequence of the variation of the preferences of the nodes, preferences that become more similar to the preferences of the nodes that have taken similar decisions in the past. At iteration $t_{0}+5$ the configuration of the network becomes 36-17-47-0 and from then on, it will be always the same. Indeed, once that the weights are not subject to great modifications, the different nodes will take always the same decision since the context does not play anymore a role in the decisions taken by the nodes. Let us mention that when we state that the configuration of the network reached at iteration $t_{0}+5$, that is $36-17-47-0$, does not change in the following iterations, we mean not only that the number of nodes taking a certain decision does not change (that is, there will be always 36 nodes choosing the first bar, 17 nodes choosing the second bar and so on) but also that the same nodes will take always the same decision. This means that, beginning from iteration $t_{0}+5$, each node $n_{h}$ will always take the same decision.

Before concluding this part, we would like to interpret the oscillations in the configurations of the network observed in Table 3a. Indeed, as we previously explained, starting from a certain iteration, the weights of the different nodes are not subject to any modification. Nevertheless, the network oscillates always between two configurations (see the configurations at the time instants $t_{0}+4$ and $t_{0}+6$ as well as the configurations at time instants $t_{0}+5$ and $t_{0}+7$ ). Let us start our analysis from the time instant $t_{0}+4$ considering the network configuration at this time instant, that is, 36-0-64-0. At this time instant, $b a r_{1}$ and $b a r_{3}$ are the most crowded, while $b a r_{2}$ and $b a r_{4}$ are empty. Due to an increase of their customers, according to Eq. (8), the evaluations got by bar $_{2}$ and bar $_{4}$ will decrease at the time instant $t_{0}+5$ rendering the two bars more appealing for the different customers that, consequently, will decide to leave bar $_{1}$ and bar $_{3}$ in favor of bar $_{2}$ and $b_{a r}$. For this reason, the configuration of the network at the time instant $t_{0}+5$ will be 3-79-0-18. Analogous reasons explain why at the next time instant $\left(t_{0}+6\right)$, the customers decide to leave $b a r_{2}$ and $b a r_{4}$ in favor, again of $b a r_{1}$ and $b a r_{3}$.

In the second part of this section we show how the structure of the network affects the dynamics of the preferences. For this purpose, we assume that the network follows the BA model (Barabási and Albert 1999), with a linear preferential attachment. In a first moment we assume that the inclinations of the nodes, as well as the starting weights and the parameter $\alpha$, are those considered in the ER network. By applying our model to this network structure we can observe the dynamics of preferences shown in Fig. 6.

We reported in Table 4 the dynamics of the preferences for the first iterations in the two different network models. As one can see, at the time instant $t_{0}$, the decisions taken by the nodes in the two networks are the same decisions taken at the time instant $t_{0}$ in the ER network, since we supposed that the nodes have initially the same starting weights. Already at iteration $t_{0}+1$, it is notable observing that the choices done by the nodes in the two networks are different. Indeed, in the ER model, 52 customers decided to go to bar $_{2}$ while, in the BA model, 59 customers decided to go to the same bar. Moreover, while the number of customers going in bars 1 and 3 is the same in the two 


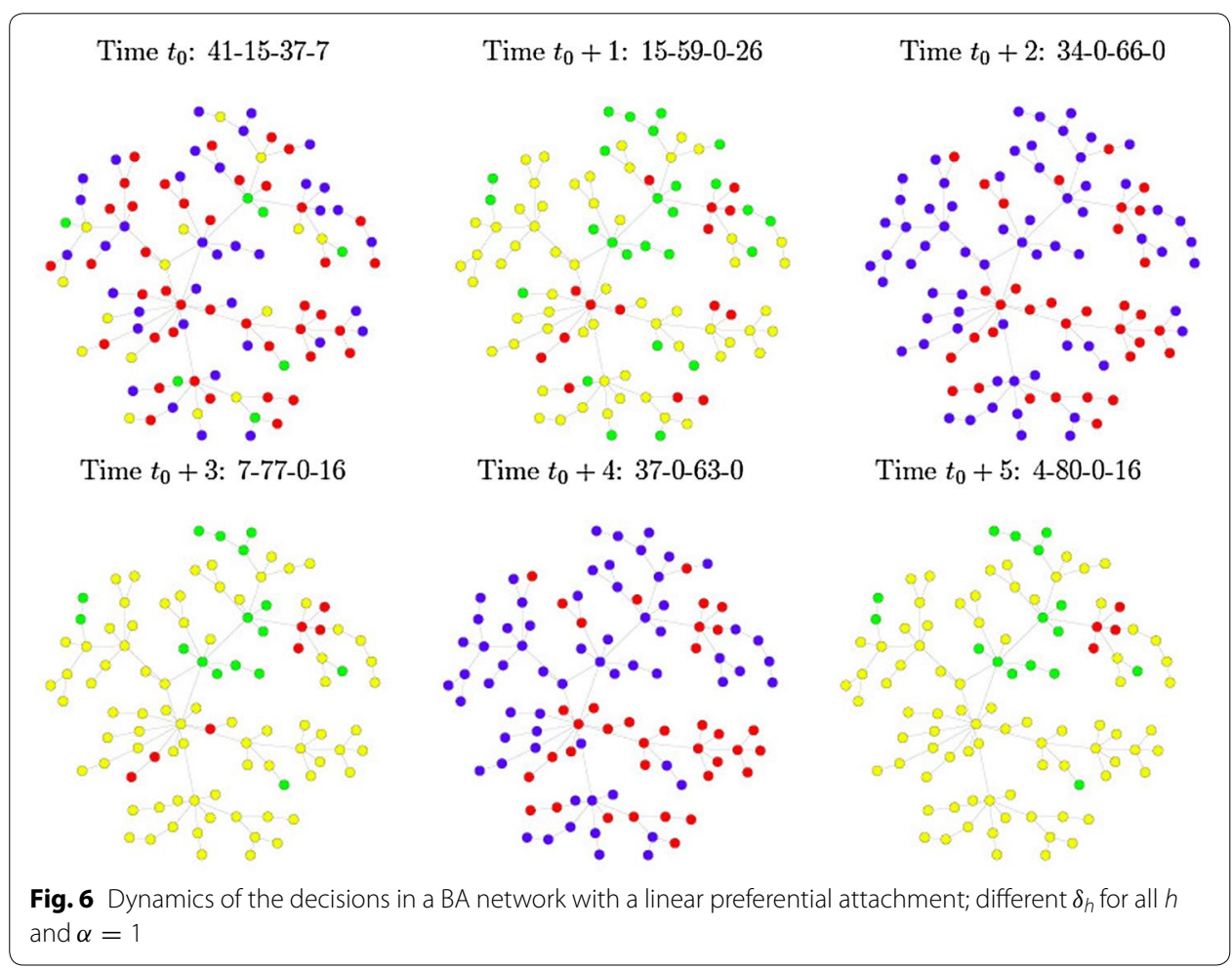

Table 4 Comparison between the dynamics of the decisions taken by the nodes in the two network models

\begin{tabular}{llll}
\hline Time & Configuration & Time & Configuration \\
\hline (a) ER model & & (b) BA model & \\
$t_{0}$ & $41-15-37-7$ & $t_{0}$ & $41-15-37-7$ \\
$t_{0}+1$ & $15-52-0-33$ & $t_{0}+1$ & $15-59-0-26$ \\
$t_{0}+2$ & $37-0-63-0$ & $t_{0}+2$ & $34-0-66-0$ \\
$t_{0}+3$ & $5-77-0-18$ & $t_{0}+3$ & $7-77-0-16$ \\
$t_{0}+4$ & $36-0-64-0$ & $t_{0}+4$ & $37-0-63-0$ \\
$t_{0}+5$ & $3-79-0-18$ & $t_{0}+5$ & $4-80-0-16$ \\
$t_{0}+6$ & $36-0-64-0$ & $t_{0}+6$ & $36-0-64-0$ \\
$t_{0}+7$ & $3-79-0-18$ & $t_{0}+7$ & $5-79-0-16$ \\
\hline
\end{tabular}

network models, the number of customers deciding to go to $b a r_{4}$ is different (33 in the ER model and 26 in the BA model). Another aspect really relevant is that, while the vector of preferences begins to oscillate between two configurations already at iterations $t_{0}+4$ and $t_{0}+5$ in the ER model, the same behavior can not be observed for the BA model. Indeed, in this model, the vector of preferences oscillates between different configurations (36-0-64-0 and 3-79-0-18 in the ER model and 5-79-0-16 and 33-067-0 in the BA model) and, moreover, these oscillations begin later than in the ER model (at iterations $t_{0}+42$ and $t_{0}+43$ instead of iterations $t_{0}+4$ and $t_{0}+5$ in the ER model). Because, as previously underlined, the main components of the network (nodes' inclinations and $\alpha$ ) are the same, the different dynamics of the preferences in the two networks 
is due to their structures and, in particular, to the number of connections of each node. This implies that while, on average, ten nodes can influence the variation of the weights of each node in the ER model, ${ }^{2}$ in the BA model the preferential attachment law implies that not all the nodes are connected to the same number of nodes.

As already done for the ER model, we show here that the variation of the main components of the model affects the dynamics of the preferences also with a different network model. To highlight the influence of nodes' inclinations on the dynamics of the preferences, we performed three different simulations in which we swapped the inclinations of ten nodes. In particular, in the first case we swapped the inclination of the same nodes already considered in the ER model. What we observed is that the dynamics of preferences in this case is very similar to that one obtained with the starting inclinations. We think that this behavior is due to the fact that the ten nodes considered in the swapping had a low degree and, consequently, they were influenced and they influenced, only a very limited number of nodes. For this reason, in the second and in the third simulations we decided to swap the inclinations of the nodes not in a random way but following a certain scheme. More precisely, in the second simulation, we swapped the inclinations of ten nodes, seven presenting an high degree and three presenting a low degree. In particular, the inclinations of five nodes having degrees 10, 8, 8, 7 and 5 have been swapped with the inclinations of five nodes having degrees 1, 7, 2, 6 and 2, in this order. This means that the inclination of the node with degree 10 has been swapped with the inclination of the node having degree 1; the inclination of the first node having degree 8 has been swapped with the inclination of the node with degree 7 , and so on. In the third simulation, instead, we swapped the inclinations of five nodes having high degree with the inclination of five nodes having low degree. In particular, the inclinations of five nodes having degrees 10, 8, 8, 7 and 7 have been swapped with the inclinations of five nodes having degrees $1,3,1,1$ and 2 .

As one can see in the middle picture and on the right picture of Fig. 7 the last two swapping of the inclinations causes a different dynamics of preferences. In particular, in the first swap, the network decisions oscillate between the configurations 0-84-0-16 and 29-0-71-0 while, in the second swap, the oscillations are between $0-81-0-19$ and 37-063-0. In these two cases, the different dynamics of the decisions is due to the fact that at least one of the nodes involved in the swapping of the inclination has a high degree and, consequently, more nodes influence its decisions than in the first swapping.

In the end, we analysed the impact of the parameter $\alpha$ on the dynamics of preferences also for the BA model. To this aim we considered two different values of $\alpha$ that are, $\alpha=1$ and $\alpha=2$. In Fig. 8, one can observe that the value of $\alpha$ affects the dynamics of preferences and, consequently, the decisions taken by the nodes in the network. In particular, at the same time instant, 23 nodes take different decisions in the two network configurations. Moreover, while in the first case the decisions oscillate between the configurations $5-79-0-16$ and 33-0-67-0, in the second one, the oscillations of decisions are between the configurations 5-56-0-39 and 42-0-58-0.

${ }^{2}$ We considered an ER model with $p=0.1$ 
Configuration considering the initial inclinations: 5-79-0-16

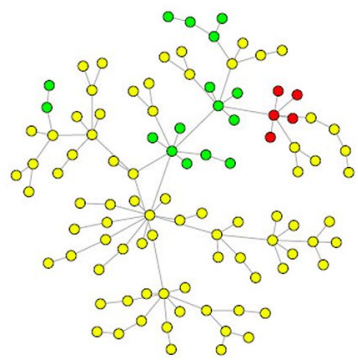

Configuration after the first swap: $0-84-0-16$

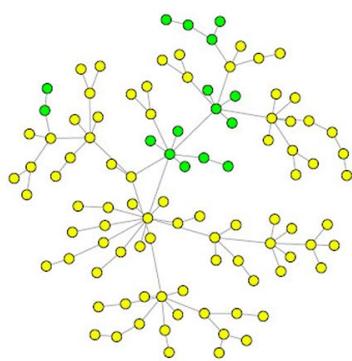

Configuration after the second swap: $0-81-0-19$

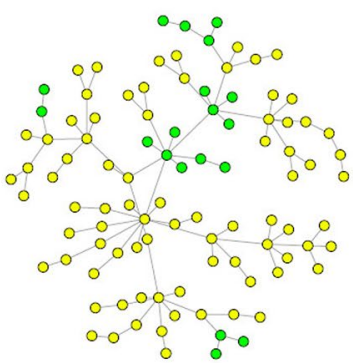

Fig. 7 Comparison of the different network configurations obtained swapping the inclination of ten nodes

Configuration of the network for $\alpha=1: 5-79-0-16 \quad$ Configuration of the network for $\alpha=2: 5-56-0-39$
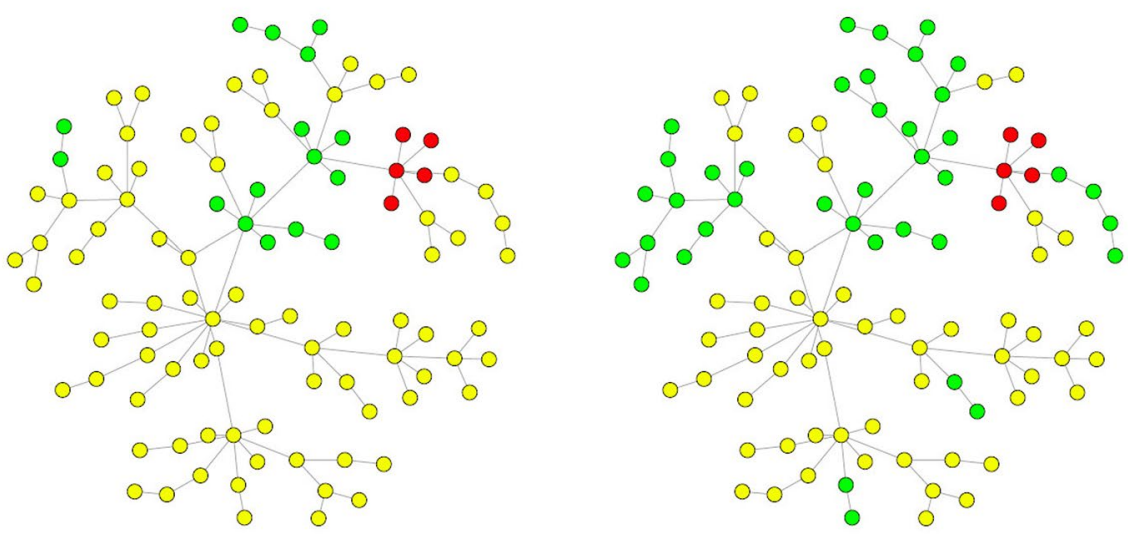

Fig. 8 Decisions taken by the nodes for two different values of $\alpha$ for the BA model

\section{Conclusions}

In this paper we proposed a new social network model in the Multiple Criteria Decision Making framework. Assuming that individual decisions are often influenced by the decisions of other individuals (López-Pintado 2008), the new model is characterized by two main novelties that are the variability of the preferences of the nodes in the considered network, and the variability of the context in which the same preferences have to be taken. On one hand, the preferences of each node are subject to the influence exercised by the nodes in the network to which it is connected. The node will be more or less subject to this influence depending on its own inclination that is represented by the parameter $\delta_{h}$. On the other hand, the variability of the context and, in particular, the context-awareness of each node, are dependent on the decisions taken by the nodes in the network at the previous instants that will also influence the decisions at the current time.

We think that the proposed model can be used to describe different dynamic decision making problems considering several network structures. To show the applicability of our model to different network structures, we applied it to the El Farol bar problem (Arthur 1994) supposing that the network follows two different models that are, the ER model (Erdös and Rényi 1959) and the BA model (Barabási and Albert 1999). Simulation 
results show that the variation of the inclination $\delta_{h}$ of each node, as well as the variability of the context represented by the parameter $\alpha$, and the number of connections between nodes in the network, affect the dynamics of the decisions in both network models. Moreover, different dynamics of the decisions have been observed in the two models as a consequence of the network's structure.

The model proposed in this paper has been simulated under simplified hypothesis but, in a more realistic scenario, there are more constraints and factors that have to be taken into account. In fact, in a social environment, not all the nodes have the same importance and, consequently, a weighted adjacency matrix could be considered to take into account this aspect. In the same direction, also the position of a node in the network, expressed by the centrality measures, can affect the dynamics of the preferences and, as a consequence, of the decisions. Furthermore, the weighted adjacency matrix, representing the importance of the links between the nodes, can vary during the time. Moreover, our model can be tested also considering a variable network structure, in which new nodes and links will be added and others removed over time, in order to study the impact of single actors in the dynamics of preferences. Finally, we believe that the presence of both an attractive and a repulsive effect on the number of individuals selecting the same goods could give interesting insights on the dynamics of such complex systems. For example, in the El Farol bar problem, it is very meaningful to investigate the interplay between the attractive effects of a larger number of customers and the consequent increase of price and uncomfortable conditions for too much crowded places.

Authors' contributions

All the six authors contributed to the development of the research activities and of the entire work. All authors read and approved the final manuscript.

\section{Author details}

${ }^{1}$ Department of Electric, Electronic and Computer Engineering, University of Catania, Viale Andrea Doria 6, Cittadella Universitaria - Ed. Polifunzionale, 95127 Catania, Italy. ${ }^{2}$ Department of Economics and Business, University of Catania, Corso Italia, 55, 95129 Catania, Italy. ${ }^{3}$ Centre of Operations Research and Logistics (CORL), Portsmouth Business School, University of Portsmouth, Richmond Building, Portland Street, Portsmouth PO1 3DE, United Kingdom.

\section{Acknowledgements}

This work has been funded by the "Programma Operativo Nazionale" Ricerca \& Competitivitá "2007-2013" within the projects "PON04a2_E SINERGREEN-RES-NOVAE" and "PON04a2_C Smart Health 2.0". Salvatore Corrente and Salvatore Greco wish also to acknowledge funding by the "FIR of the University of Catania BCAEA3 New developments in Multiple Criteria Decision Aiding (MCDA) and their application to territorial competitiveness"

\section{Competing interests}

The authors declare that they have no competing interests.

Received: 6 November 2015 Accepted: 26 February 2016

Published online: 09 March 2016

\section{References}

Abowd GD, Dey AK, Brown PJ, Davies N, Smith M, Steggles P. Towards a better understanding of context and contextawareness. In: Gellersen HW, editor. Handheld and Ubiquitous Computing, LNCS 1707. Berlin: Springer; 1999. p. 304-7.

Ackert L, Deaves R. Behavioral finance: psychology, decision-making, and markets. South-Western: Cengage Learning; 2009.

Aggarwal CC. Social network data analytics. Berlin: Springer; 2011.

Ahn S, Kim D. Proactive context-aware sensor networks. In: Römer K, Karl H, Mattern F, editors. Wireless sensor networks, LNCS 3868. Berlin: Springer; 2006. p. 38-53.

Anagnostopoulos A, Becchetti L, Bordino I, Leonardi S, Mele I, Sankowski P. Stochastic query covering for fast approximate document retrieval. ACM Trans Inf Syst. 2015;33(3):11-1-11-35.

Arthur WB. Inductive reasoning and bounded rationality. Am Econ Rev 1994;84(2):406-11.

Asavathiratham C, Roy S, Lesieutre B, Verghese G. The influence model. IEEE Control Syst. 2001;21(6):52-64.

Avery C, Zemsky P. Multidimensional uncertainty and herd behavior in financial markets. Am Econ Rev. 1998;88:724-48. 
Banerjee AV. A simple model of herd behavior. Q J Econ. 1992;107:797-817.

Barabási AL, Albert R. Emergence of scaling in random networks. Science. 1999;286(5439):509-12.

Barjis J, Gupta A, Sharda R. Knowledge work and communication challenges in networked enterprises. Inf Syst Frontiers. 2011;13(5):615-9.

Bikhchandani S, Hirshleifer D, Welch I. A theory of fads, fashion, custom, and cultural change as informational cascades. J Polit Econ. 1992;100:992-1026.

Bottazzi D, Montanari R, Toninelli A. Context-aware middleware for anytime, anywhere social networks. IEEE Intell Syst. 2007;22:23-32.

Brans JP, Vincke P. A preference ranking organisation method: the PROMETHEE method for MCDM. Manag Sci. 1985;31(6):647-56.

Brown PJ, Bovey JD, Chen X. Context-awareness applications: from the laboratory to the marketplace. IEEE Pers Commun. 1997:4:58-64.

Brunnermeier MK. Asset pricing under asymmetric information: bubbles, crashes, technical analysis, and herding. Oxford: Oxford University Press; 2001.

Cacioppo JT, Fowler JH, Christakis NA. Alone in the crowd: the structure and spread of loneliness in a large social network. J Personal Soc Psychol. 2009;97(6):977.

Camerer CF, Loewenstein G, Rabin M. Advances in Behavioral Economics. New York: Princeton University Press; 2003.

Chen G, Kotz D, et al (2000) A survey of context-aware mobile computing research. Technical Report TR2000-381, Department of Computer Science, Dartmouth College.

Christakis NA, Fowler JH. The spread of obesity in a large social network over 32 years. N Engl J Med. 2007;357(4):370-9.

Christakis NA, Fowler JH. The collective dynamics of smoking in a large social network. N Engl J Med. 2008:358(21):2249-58

Christakis NA, Fowler JH. Social contagion theory: examining dynamic social networks and human behavior. Stat Med. 2013;32(4):556-77.

Cioffi-Revilla C. Introduction to computational social science: principles and applications. Berlin: Springer, Science \& Business Media; 2013

Corrente S, Greco S, Kadziński M, Słowiński R. Robust ordinal regression. New York: Wiley Encyclopedia of Operational Research; 2014.

Di Stefano A, Scatà M, La Corte A, Liò P, Catania E, Guardo E, Pagano S. Quantifying the role of homophily in human cooperation using multiplex evolutionary game theory. PloS One. 2015;10(10):e0140646. doi:10.1371/journal. pone.0140646.

Easley D, Kleinberg J. Networks, crowds, and markets: reasoning about a highly connected world. Cambridge: Cambridge University Press; 2010.

Erdös P, Rényi A. On random graphs I. Publ Math Debrecen. 1959;6:290-7.

Figueira J, Greco S, Ehrgott M. Multiple criteria decision analysis: state of the art surveys. Berlin: Springer; 2005.

Figueira JR, Greco S, Słowiński R, Roy B. An overview of ELECTRE methods and their recent extensions. J Multicriteria Decis Anal. 2013;20:61-85.

Fortunato S. Community detection in graphs. Phys Rep. 2010;486(3):75-174

Giacchi E, Di Stefano A, La Corte A, Scatà M (2014) A dynamic context-aware multiple criteria decision making model in social networks. In: 2014 International conference on information society (i-Society). IEEE, pp 157-162.

Gigerenzer G, Goldstein DG. Reasoning the fast and frugal way: models of bounded rationality. Psychol Rev. 1996:103(4):650.

Grabisch M, Rusinowska A. A model of influence in a social network. Theory Decis. 2010;69(1):69-96.

Grabisch M, Rusinowska A. A model of influence with an ordered set of possible actions. Theory Decis. 2010;69(4):635-56.

Greco S, Matarazzo B, Słowiński R. Rough sets theory for multicriteria decision analysis. Eur J Oper Res. 2001;129(1):1-47.

Greco S, Mousseau V, Słowiński R. Ordinal regression revisited: multiple criteria ranking using a set of additive value functions. Eur J Oper Res. 2008;191(2):416-36.

Guermah H, Fissaa T, Hafiddi H, Nassar M, Kriouile, A. Context modeling and reasoning for building context aware services. In: 2013 ACS international conference on computer systems and applications (AICCSA); 2013. p. 1-7. IEEE.

Hämäläinen RP, Luoma J, Saarinen E. On the importance of behavioral operational research: the case of understanding and communicating about dynamic systems. Eur J Oper Res. 2013;228(3):623-34

Hoede C, Bakker RR. A theory of decisional power. J Math Sociol. 1982;8(2):309-22.

Jacquet-Lagrèze E, Siskos Y. Preference disaggregation: 20 years of MCDA experience. Eur J Oper Res. 2001;130(2):233-45.

Keeney R, Raiffa H. Decisions with multiple objectives: preferences and value tradeoffs. New York: Wiley; 1993.

Kempe D, Kleinberg JM, Tardos E. Maximizing the spread of influence through a social network. Theory Comput. 2015;11:105-47.

Korhonen P, Wallenius J. Behavioral issues in MCDM: neglected research questions. In: Climaco J, editor. Multicriteria analysis. Springer, Berlin;1997. p. 412-22.

Korhonen P, Moskowitz H, Wallenius J. Choice behavior in interactive multiple-criteria decision making. Ann Oper Res. 1990;23(1):161-79.

Lewis K, Gonzalez M, Kaufman J. Social selection and peer influence in an online social network. Proc Natl Acad Sci. 2012;109(1):68-72

Liu W, Li X, Huang D. A survey on context awareness. In: Proceedings of the 2011 international conference on computer science and service systems (CSSS); 2011. p. 144-7.

López-Pintado D. Diffusion in complex social networks. Games Econ Behav. 2008;62(2):573-90.

McPherson M, Smith-Lovin L, Cook JM. Birds of a feather: homophily in social networks. Annu Rev Sociol; 2001. p. 415-44

Pachidi S, Spruit M, Van De Weerd I. Understanding users' behavior with software operation data mining. Comput Hum Behav. 2014;30:583-94.

Pentland A. Social physics: How good ideas spread-the lessons from a new science. USA: Penguin; 2014.

Roy B. Multicriteria methodology for decision aiding. Dordrecht: Kluwer; 1996. 
Rusinowska A, de Swart H. Generalizing and modifying the Hoede-Bakker index. In: de Swart H, Osloska E, Schmidt G,

Roubens M, editors. Theory and applications of relational structures as knowledge Instruments, No 2. Lecture Notes in Artificial Intelligence LNAI 4342. Springer, Heidelberg, Germany. 2006. p. 60-88.

Schelling TC. Models of segregation. Am Econ Rev. 1969;59(2):488-93.

Schilit BN, Theimer MM. Disseminating active map information to mobile hosts. IEEE Netw. 1994;8(5):22-32.

Schilit BN, Adams N, Want R. Context-aware computing applications. In: Proceedings of the IEEE workshop on mobile computing systems and applications; 1994. p. 85-90.

Snowdon D, Grasso A. Providing context awareness via a large screen display. In: Proceedings of the CHI 2000 workshop on "The What, Who, Where, When, Why and How of Context Awareness"; 2000.

Tavana M, Keramatpour M, Santos-Arteaga FJ, Ghorbaniane E. A fuzzy hybrid project portfolio selection method using data envelopment analysis, TOPSIS and integer programming. Expert Syst Appl. 2015;42(22):8432-44.

Wang Y, Ruhe G. The cognitive process of decision making. J Cogn Inf Nat Intell. 2007;1:73-85.

Wasserman S, Faust K. Social network analysis: methods and applications, vol. 8. Cambridge: Cambridge University Press; 1994.

Watts DJ, Strogatz SH. Collective dynamics of'small-world'networks. Nature. 1998;393(6684):440-2.

\section{Submit your manuscript to a SpringerOpen ${ }^{\circ}$ journal and benefit from:}

- Convenient online submission

- Rigorous peer review

- Immediate publication on acceptance

- Open access: articles freely available online

- High visibility within the field

- Retaining the copyright to your article 